B. Le Stum and A. Quirós

Nagoya Math. J.

Vol. 191 (2008), 79-110

\title{
THE FILTERED POINCARÉ LEMMA IN HIGHER LEVEL \\ (WITH APPLICATIONS TO ALGEBRAIC GROUPS)
}

\author{
BERNARD LE STUM AND ADOLFO QUIRÓS
}

\begin{abstract}
We show that the Poincaré lemma we proved elsewhere in the context of crystalline cohomology of higher level behaves well with regard to the Hodge filtration. This allows us to prove the Poincaré lemma for transversal crystals of level $m$. We interpret the de Rham complex in terms of what we call the Berthelot-Lieberman construction and show how the same construction can be used to study the conormal complex and invariant differential forms of higher level for a group scheme. Bringing together both instances of the construction, we show that crystalline extensions of transversal crystals by algebraic groups can be computed by reduction to the filtered de Rham complexes. Our theory does not ignore torsion and, unlike in the classical case $(m=0)$, not all invariant forms are closed. Therefore, close invariant differential forms of level $m$ provide new invariants and we exhibit some examples as applications.
\end{abstract}

\section{Introduction}

In a series of articles, starting with [9] and [10], we have been using the partial divided powers of Berthelot to study the geometry of algebraic varieties of positive characteristic. This gives new insight into the $p$-adic cohomological theories. Unlike other works on the subject ([7], [13] and [5]), we do not use crystalline cohomology of higher level as a tool to obtain results in rigid cohomology and, in particular, we do not ignore torsion. In fact, torsion is very rich in this theory and provides new invariants that help understand the geometry of algebraic varieties. For example, we will show how the sheaf of closed invariant differential forms of higher level can tell you exactly where the supersingular locus of a family of elliptic curves is.

Received July 6, 2006.

Revised June 18, 2007.

2000 Mathematics Subject Classification: Primary 14F30; Secondary 14F40, 14L15.

Our collaboration was made possible by the MCRTN Arithmetic Algebraic Geometry (MRTN-CT-2003-504917) of the European Union. The second author was partially supported by MCYT (Spain) project GALAR (MTM2006-10548) and Madrid Region project TENU (CCG06-UAM/ESP-0477). 
Following A. Ogus in [11], we introduced in [9] the notion of transversal crystal of higher level. Although we could slightly improve on some of Ogus' results, progress was hampered by the lack of de Rham techniques for computing exactly crystalline cohomology in higher level. A natural answer to this problem was provided in [10], where we developed ideas of P. Berthelot, introducing the de Rham complex of higher level and proving the exact Poincaré lemma. In the present article, we extend the Poincaré lemma of higher level to transversal crystals by paying close attention to filtrations. This is used to give a precise description of the group of extensions of a transversal crystal by a commutative group scheme. In a forthcoming article, we want to use these results to show that Dieudonné crystals of higher level are transversal.

After reviewing in Section 1 a few results on filtrations, and especially fixing terminology about filtered derived categories, in Section 2 we verify that the formal Poincaré lemma in higher level behaves as expected with respect to filtrations. In doing so, we reinterpret the de Rham and linearized de Rham complexes introduced in [10] as particular cases of what we call a Berthelot-Lieberman complex. This general construction will be used in Section 4 to define the conormal complex of higher level of a group scheme. But first we prove the filtered Poincaré lemma for transversal crystals. This is done in Section 3 and requires careful attention to the behavior of the filtrations all along the process. In Section 4, which is completely independent of the preceding one, we study the relation between the conormal sheaf of higher level and invariant differential forms of higher level. Unlike in the classical case (level 0), not all invariant forms are closed. Actually, the module of closed invariant forms is isomorphic to the first cohomology group of the conormal complex. We present concrete examples, including the Legendre family of elliptic curves, and give the relation with de Rham cohomology of higher level in the case of abelian schemes. Section 5 brings together the two previous sections. More precisely, we show that crystalline extensions of transversal crystals by abelian groups can be computed by reduction to filtered de Rham complexes. As an application, we show that the extension group of the partial divided power ideal by a smooth abelian group is nothing other than a lifting of the module of closed invariant differentials of higher level.

Most results here are inspired by theorems that have been well known for a long time in the case of usual divided powers and classical crystalline 
cohomology. In particular, this work owes much to P. Berthelot, L. Breen, L. Illusie, W. Messing and A. Ogus.

Many thanks to the referee who pointed out several mistakes and encouraged us to improve the presentation of this article.

\section{Conventions}

Starting at Section 2, we let $p$ be a prime, $m \in \mathbf{N}$ and, unless $m=0$, all schemes are assumed to be $\mathbf{Z}_{(p)}$-schemes.

\section{$\S 1$. Generalities about filtrations}

Concerning filtrations, we use the terminology of [6], 1.1. In particular, we will only consider filtrations of type $\mathbf{Z}$ in additive categories. Also, filtration will always mean decreasing filtration.

Definition 1.1. A filtration $\mathrm{Fil}^{\bullet}$ on an object $M$ is effective if $\mathrm{Fil}^{0} M=M$.

Unless otherwise specified, we will only consider effective decreasing filtrations. Note that the filtration induced on a subquotient (cf. [6], 1.1.10) by an effective filtration is still effective. One can also check that the image of an effective filtration by a semi-exact (meaning left or right exact) multiadditive functor (cf. [6], 1.1.12) is still effective. This applies in particular to the tensor product filtration.

Definition 1.2. The trivial (effective) filtration on an object $M$ is given by

$$
\mathrm{Fil}^{k} M= \begin{cases}M & \text { if } k \leq 0 \\ 0 & \text { if } k>0\end{cases}
$$

In [6] (Definition 1.3.6), filtered quasi-isomorphisms are only defined for so-called biregular filtrations. This definition does not generalize well. As in [11], Section 4.4, we will need a more restrictive notion.

Definition 1.3. A morphism of filtered complexes $M^{\bullet} \rightarrow N^{\bullet}$ is a true filtered quasi-isomorphism if, for each $k \in \mathbf{Z}$, the induced morphism $\mathrm{Fil}^{k} M^{\bullet} \rightarrow \mathrm{Fil}^{k} N^{\bullet}$ is a quasi-isomorphism. A filtered homotopy is a homotopy $s: M^{\bullet} \rightarrow N^{\bullet}$ such that

$$
\forall k \in \mathbf{Z}, \quad \forall n \in \mathbf{Z}, \quad s\left(\mathrm{Fil}^{k} M^{n}\right) \subset \mathrm{Fil}^{k} N^{n-1} .
$$


Two morphisms of filtered complexes $f, g: M^{\bullet} \rightarrow N^{\bullet}$ are homotopic if there exists a filtered homotopy $h: M^{\bullet} \rightarrow N^{\bullet}$ such that $g$ is homotopic to $f$ with respect to $h$.

As explained in [11], page 80, the above notions of filtered homotopy and true filtered quasi-isomorphisms are suitable to define the filtered derived category of a Grothendieck category, e.g. a category of modules. Moreover, any left exact additive functor $F$ between such categories gives rise to a filtered derived functor and this construction is completely compatible with the non-filtered situation in the sense that we always have

$$
R F\left(\mathrm{Fil}^{k} M^{\bullet}\right)=\mathrm{Fil}^{k} R F M^{\bullet} \text {. }
$$

In particular, there exists a canonical spectral sequence

$$
E_{1}^{i, j}=R^{i+j} F\left(G r^{i} M^{\bullet}\right) \Rightarrow R^{n} F\left(M^{\bullet}\right)
$$

that endows, for each $n \in \mathbf{Z}, R^{n} F\left(M^{\bullet}\right)$ with a canonical filtration.

We now need to recall some definitions and results from [11] and [9] on transversal filtrations.

Definition 1.4. A ring filtration on a ring $A$ is an effective filtration by ideals $I^{(k)}$ such that $I^{(k)} I^{(l)} \subset I^{(k+l)}$. A filtered $A$-module $\left(M\right.$, Fil $\left.{ }^{\bullet}\right)$ is transversal (resp. almost transversal) to $I^{(\bullet)}$ if for each $k \in \mathbf{Z}$,

$$
I^{(1)} M \cap \mathrm{Fil}^{k} M=(\text { resp. } \subset) \sum_{i+j=k, i>0} I^{(i)} \mathrm{Fil}^{j} .
$$

If $\left(M, \mathrm{Fil}^{\bullet}\right)$ is a filtered $A$-module, the saturation of the filtration with respect to $I^{(\bullet)}$ is the tensor product filtration $\overline{\text { Fil }} \bullet$ on $M$ under the identification $A \otimes_{A} M=M$.

When $A$ is filtered by the powers of an ideal $I$, we simply say transversal (resp. almost transversal, resp. saturation with respect) to $I$. Note that a module filtration is always transversal to 0 and that, if a filtration is almost transversal, then its saturation is transversal.

Definition 1.5. The trivial transversal filtration on an $A$-module $M$ is the saturation of the trivial filtration.

Note that the trivial transversal filtration is given by

$$
\overline{\mathrm{Fil}}^{k} M= \begin{cases}M & \text { if } k \leq 0 \\ I^{(k)} M & \text { if } k>0\end{cases}
$$




\section{$\S 2 . \quad$ The formal filtered Poincaré lemma}

The aim of this section is to give a filtered version of Theorem 3.3 of [10]. We first recall some properties of $m$-PD-envelopes and introduce the notion of Berthelot-Lieberman complex.

If $X \hookrightarrow Y$ is an immersion of schemes, then we will denote by $P_{X m}(Y)$ its divided power envelope of level $m$. We will write $\mathcal{P}_{X m}(Y)$ for the structural sheaf and $\mathcal{I}_{X m}(Y)$ for the $m$-PD-ideal. We will use a superscript $n$ to denote the same objects modulo $\mathcal{I}_{X m}^{\{n+1\}}(Y)$. If $X$ is an $S$-scheme, we will denote by $P_{X / S m}(r)$ the $m$-PD envelope of the diagonal embedding of $X$ in $X^{r+1}$ and modify all other notations accordingly.

The notion of $m$-PD envelope is functorial in the sense that any commutative diagram

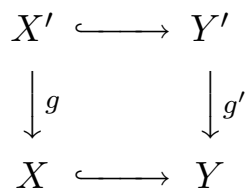

canonically extends to

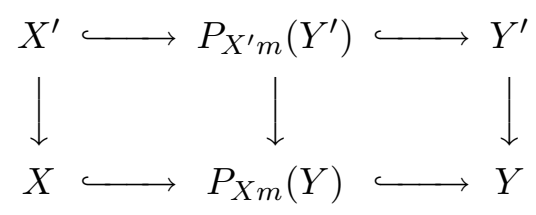

We recall the following fundamental results of Berthelot:

Proposition 2.1. Let $f: X \hookrightarrow Y$ be an immersion of schemes with a smooth retraction $h: Y \rightarrow X$. We use $h$ to endow $\mathcal{P}_{X m}^{n}(Y)$ and $\mathcal{P}_{X m}(Y)$ with the structure of an $\mathcal{O}_{X}$-algebra. Then,

1. For all $n, \mathcal{P}_{X m}^{n}(Y)$ is a locally free $\mathcal{O}_{X}$-module of finite rank. Actually, $\mathcal{P}_{X m}(Y)$ itself is locally free when $Y$ is uniformly killed by a power of $p$.

2. Let

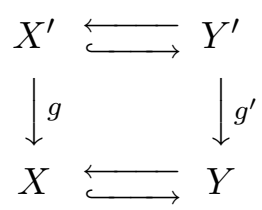


be a be cartesian diagram (both ways). Then, we have for all $n$,

$$
g^{*} \mathcal{P}_{X m}^{n}(Y) \simeq \mathcal{P}_{X^{\prime} m}^{n}\left(Y^{\prime}\right)
$$

and even

$$
g^{*} \mathcal{P}_{X m}(Y) \simeq \mathcal{P}_{X^{\prime} m}\left(Y^{\prime}\right)
$$

if $Y$ is uniformly killed by a power of $p$.

Proof. This follows from Propositions 1.4.6 and 1.5.3 of [2].

Let $X \hookrightarrow Y(\bullet)$ be an immersion of a scheme into a simplicial scheme. In other words, we are given a family of immersions of $X \hookrightarrow Y(r)$ compatible with the differentials $d_{i}$ and the degeneracy arrows $s_{i}$ of $Y(\bullet)$. Taking $m$-PD envelopes gives rise to a simplicial scheme $P_{X m}(Y \bullet)$ from which we derive a complex $\left(\mathcal{P}_{X m}(Y \bullet), \mathcal{I}_{X m}^{\{k\}}(Y \bullet)\right)$ of filtered rings. We then consider as in [8] the normalization of $\mathcal{P}_{X m}(Y \bullet)$ which is the subcomplex of ideals defined by $N \mathcal{P}_{X m}(Y r):=\bigcap$ ker $s_{i}^{*}$. Finally, we are interested in the quotient of $N \mathcal{P}_{X m}(Y \bullet)$ by the differential subalgebra generated by the ideal $\mathcal{I}_{X m}^{\left\{p^{m}+1\right\}}(Y 1)$ of $\mathcal{P}_{X m}(Y 1)$, which we write $\Omega_{X m}^{\bullet}(Y)$.

Definition 2.2. The complex $\Omega_{X m}^{\bullet}(Y)$ is the Berthelot-Lieberman complex of $X \hookrightarrow Y(\bullet)$. The Hodge filtration on $\Omega_{X m}^{\bullet}(Y)$ is the filtration $\mathrm{Fil}_{H}^{k}$ induced by the $m$-PD-filtration $\mathcal{I}_{X m}^{\{k\}}(Y \bullet)$ of $\mathcal{P}_{X m}(Y \bullet)$.

\section{DEFINITION 2.3.}

1. In a category with products, the product simplicial object $X^{\text {prod }}(\bullet)$ of an object $X$ is defined by $X^{\text {prod }}(r)=X^{r+1}$,

$$
d_{i}\left(x_{1}, \ldots, x_{r+2}\right)=\left(x_{1}, \ldots, x_{i}, x_{i+2}, \ldots, x_{r+2}\right) \text { for } i=0,1, \ldots, r+1
$$

and

$$
\begin{aligned}
s_{i}\left(x_{1}, \ldots, x_{r}\right)=\left(x_{1}, \ldots, x_{i}, x_{i+1}, x_{i+1}, x_{i+2}, \ldots,\right. & \left.x_{r+2}\right) \\
& \text { for } i=0,1, \ldots, r .
\end{aligned}
$$

2. If $X(\bullet)$ is a simplicial object in any category, the shifted simplicial object is defined by

$$
X^{+}(r)=X(r+1), \quad d_{i}^{+}=d_{i+1}, \quad s_{i}^{+}=s_{i+1} .
$$


We can now reformulate some definitions from Section 1 of [10].

Let $S$ be a scheme with $p$ locally nilpotent and $X$ an $S$-scheme.

Definition 2.4. The Berthelot-Lieberman complex $\Omega_{X / S m}^{\bullet}$ of the diagonal embedding $X \hookrightarrow(X / S)^{\operatorname{prod}}(\bullet)$, is the de Rham complex of level $m$. The Berthelot-Lieberman complex $L_{X}\left(\Omega_{X / S m}^{\bullet}\right)$ of the shifted simplicial scheme $(X / S)^{\text {prod }+}(\bullet)$ is the linearized de Rham complex of level $m$.

Note that $L_{X}\left(\Omega_{X / S m}^{\bullet}\right)$ is what we called $\Omega_{P / S m}^{\bullet}$ in [10].

For the rest of this section, we assume that $X$ is a smooth scheme over $S$.

Remarks 2.5 .

1. Recall from Section 1 of [10] that, if we have local coordinates $t_{1}, \ldots, t_{n}$ on $X$ and, as usual, we set $\tau_{i}:=1 \otimes t_{i}-t_{i} \otimes 1$, then $\mathcal{P}_{X / S m}(r)$ is a free $\mathcal{O}_{X}$-module on generators $\tau^{\left\{J_{1}\right\}} \otimes \cdots \otimes \tau^{\left\{J_{r}\right\}}$ with $\left|J_{i}\right| \geq 0$ (we use the standard multiindex convention). Using Proposition 1.5.3 of [2], one easily checks that $\mathcal{I}_{X / S m}^{\{k\}}(r)$ is generated by the $\tau^{\left\{J_{1}\right\}} \otimes \cdots \otimes \tau^{\left\{J_{r}\right\}}$ with $\left|J_{1}\right|+\cdots+\left|J_{r}\right| \geq k$.

2. Now, if as usual again, $d t_{i}$ denotes the image of $\tau_{i}$ in $\Omega_{X / S m}^{1}$, we know that $\Omega_{X / S m}^{1}$ is a free $\mathcal{O}_{X}$-module on generators $(d t)^{J}$ with $0<|J| \leq$ $p^{m}$. As we discussed in Section 1 of [10], for $r>1$, even if $\Omega_{X / S m}^{r}$ is generated by the $(d t)^{J_{1}} \otimes \cdots \otimes(d t)^{J_{r}}$ with $0<\left|J_{i}\right| \leq p^{m}$, there are some relations among these generators. Actually, $\Omega_{X / S m}^{\bullet}$ is the quotient of the tensor algebra $T^{\bullet}\left(\Omega_{X / S m}^{1}\right)$ by the relations

$$
\sum_{0<V<J}\left\langle\begin{array}{c}
J \\
V
\end{array}\right\rangle(d t)^{J-V} \otimes(d t)^{V}=0 .
$$

By definition, we see that the Hodge filtration $\mathrm{Fil}_{H}^{k} \Omega_{X / S m}^{r}$ is generated by the

$$
(d t)^{J_{1}} \otimes \cdots \otimes(d t)^{J_{r}} \text { with } 0<\left|J_{i}\right| \leq p^{m} \text { and }\left|J_{1}\right|+\cdots+\left|J_{r}\right| \geq k .
$$

In particular, $\operatorname{Fil}_{H}^{k}=0$ for $k>r p^{m}$. 
3. Similarly, $L_{X}\left(\Omega_{X / S m}^{r}\right)$ is generated as $\mathcal{P}_{X / S m}(1)$-module by the $(d \tau)^{J_{1}} \otimes \cdots \otimes(d \tau)^{J_{r}}$ with $0<\left|J_{i}\right| \leq p^{m}$, and $\mathrm{Fil}_{H}^{k} L_{X}\left(\Omega_{X / S m}^{r}\right)$ is the $\mathcal{O}_{X}$-submodule generated by the $\tau^{\{I\}}(d \tau)^{J_{1}} \otimes \cdots \otimes(d \tau)^{J_{r}}$ with $|I|+\left|J_{1}\right|+\cdots+\left|J_{r}\right| \geq k$.

4. The Hodge filtration on $\Omega_{X / S m}^{r}$ is a filtration where, for each $k \geq 0$, both $\mathrm{Fil}^{k} \Omega_{X / S m}^{r}$ and $\mathrm{Gr}^{k} \Omega_{X / S m}^{r}$ are locally free. To see this, one proceeds as in the proof of Proposition 1.4 in [10]. First of all, the question is local. Then, the process consists in writing some of the $(d t)^{J_{1}} \otimes \cdots \otimes(d t)^{J_{r}}$ with $\left|J_{1}\right|+\cdots+\left|J_{r}\right|=k$ in term of the others using the relations $(*)$ which is possible because $|J-V|+|V|=|J|$ for all $V$.

5. Note that when $X$ is smooth, the de Rham complex of level 0 is the usual de Rham complex with its Hodge filtration. The reason is that the generators of $\Omega_{X / S 0}^{r}$ are the $d t_{i_{1}} \otimes \cdots \otimes d t_{i_{r}}$ and the relations $(*)$ become simply

$$
d t_{i} \otimes d t_{i}=0 \quad \text { and } \quad d t_{i} \otimes d t_{j}+d t_{j} \otimes d t_{i}=0
$$

Recall that if $\mathcal{F}$ is any $\mathcal{D}_{X / S}^{(m)}$-module, we can form the de Rham complex

$$
\mathcal{F} \otimes_{\mathcal{O}_{X}} \Omega_{X / S m}^{\bullet}
$$

of $\mathcal{F}$. In particular, we have the m-connection $\mathcal{F} \rightarrow \mathcal{F} \otimes_{\mathcal{O}_{X}} \Omega_{X / S m}^{1}$. For further use, recall also that the relative de Rham cohomology of level $m$ of $\mathcal{F}$ is

$$
\mathcal{H}_{d R m}^{n}(X, \mathcal{F}):=\mathbf{R}^{n} p_{X *} \mathcal{F} \otimes_{\mathcal{O}_{X}} \Omega_{X / S m}^{\bullet}
$$

where $p_{X}: X \rightarrow S$ is the structural map.

Concerning Griffiths transversality, we refer to Section 2.2 of [9]. Let us just recall that a filtration $\mathrm{Fil}^{\bullet}$ on a $\mathcal{D}_{X / S^{-}}^{(m)}$-module $\mathcal{F}$ is a filtration by $\mathcal{O}_{X}$-submodules and that it is said Griffiths transversal if we always have

$$
\mathcal{D}_{X / S j}^{(m)} \mathrm{Fil}^{k} \mathcal{F} \subset \mathrm{Fil}^{k-j} \mathcal{F}
$$

Proposition 2.6. A filtered $\mathcal{D}_{X / S^{-m o d u l e}}^{(m)}$ is Griffiths transversal if and only if the m-connection is compatible with the filtrations. 
Proof. This is a local question and, if we have local coordinates, the $m$-connection on $\mathcal{F}$ is given by

$$
s \longmapsto \sum_{0<|J| \leq p^{m}} \partial^{[J]} s \otimes(d t)^{J} .
$$

If the filtration is Griffiths transversal and $s \in \mathrm{Fil}^{k} \mathcal{F}$, we have

$$
\partial^{[J]} s \otimes(d t)^{J} \in \mathrm{Fil}^{k-|J|} \mathcal{F} \otimes \mathrm{Fil}^{|J|} \Omega_{X / S m}^{1} .
$$

Since both the filtration and the graded pieces are locally free, we have

$$
\mathrm{Fil}^{k-|J|} \mathcal{F} \otimes \mathrm{Fil}^{|J|} \Omega_{X / S m}^{1} \subset \operatorname{Fil}^{k}\left(\mathcal{F} \otimes \Omega_{X / S m}^{1}\right) .
$$

It follows that the connection preserves the filtrations.

Conversely, if the $m$-connection is compatible with the filtrations and $s \in \mathrm{Fil}^{k} \mathcal{F}$, then, necessarily, for all $i=1, \ldots, n$ and $j \leq m$, we have

$$
\partial_{i}^{\left[p^{j}\right]} s \otimes(d t)^{p^{j}} \in \mathrm{Fil}^{k}\left(\mathcal{F} \otimes \Omega_{X / S m}^{1}\right)
$$

so that, necessarily, $\partial_{i}^{\left[p^{j}\right]} s \in \mathrm{Fil}^{k-\left|p^{j}\right|} \mathcal{F}$ and by Remark 2.2.2 (ii) of [9], this is sufficient for Griffiths transversality.

Since the differential on a de Rham complex can be described by the Leibnitz rule, we have the following:

Corollary 2.7. If $\mathcal{F}$ is a filtered $\mathcal{D}_{X / S}^{(m)}$-module and if, for each $r$, $\mathcal{F} \otimes \Omega_{X / S m}^{r}$ is endowed with the tensor product filtration, then $\mathcal{F}$ is Griffiths transversal if and only if the de Rham complex of $\mathcal{F}$ is a filtered complex.

LEMMA 2.8. For all $r$, if we endow $\Omega_{X / S m}^{r}$ and $L_{X}\left(\Omega_{X / S m}^{r}\right)$ with their Hodge filtration and $\mathcal{P}_{X / S m}(1)$ with its $m$-PD-filtration, we have an isomorphism of filtered modules

$$
L_{X}\left(\Omega_{X / S m}^{r}\right)=\mathcal{P}_{X / S m}(1) \otimes_{\mathcal{O}_{X}} \Omega_{X / S m}^{r}
$$

Moreover, the filtration on $\mathcal{P}_{X / S m}(1) \otimes \mathcal{O}_{X} \Omega_{X / S m}^{r}$ is the saturation with respect to $\mathcal{I}_{X / S m}^{\{\bullet \bullet}(1)$ of the inverse image by $\mathcal{P}_{X / S m}(1) \rightarrow \mathcal{O}_{X}$ of the Hodge filtration on $\Omega_{X / S m}^{r}$. 
Proof. Since we assume that $X$ is smooth over $S$, then as mentioned in Section 1.5 of [10], $\mathcal{P}_{X / S m}^{+}(r)$ is canonically isomorphic as filtered algebra to $\mathcal{P}_{X / S m}(1) \otimes_{\mathcal{O}_{X}} \mathcal{P}_{X / S m}(r)$. The assertion then follows from the functoriality of our construction. The second assertion is local in nature and follows directly from the local description of our filtrations in Remark 2.5.3.

Proposition 2.9. The Hodge filtration on $L_{X}\left(\Omega_{X / S m}^{\bullet}\right)$ is transversal to $\mathcal{I}_{X / S m}^{\{\bullet\}}(1)$.

Proof. The morphism of filtered rings $\left(\mathcal{P}_{X / S m}(1), \mathcal{I}_{X / S m}^{\{\bullet \bullet}(1)\right) \rightarrow\left(\mathcal{O}_{X}, 0\right)$ obviously satisfies the assumptions of Proposition 1.1 .8 of [9]. Since any filtration is transversal to the 0-ideal, we see that the inverse image of the Hodge filtration is almost transversal and it follows that its saturation is transversal. Our assertion now results from Lemma 2.8.

The following is a generalization of Theorem 3.3 of [10].

Proposition 2.10. If $\mathcal{O}_{X}$ is endowed with the trivial filtration and $L_{X}\left(\Omega_{X / S m}^{\bullet}\right)$ with the Hodge filtration, the canonical map

$$
\mathcal{O}_{X} \longrightarrow L_{X}\left(\Omega_{X / S m}^{\bullet}\right)
$$

is a true filtered quasi-isomorphism. More precisely, locally on $X$, it is a filtered homotopy equivalence.

Proof. The proof works exactly as in Theorem 3.3 of [10] once one notices that the homotopy of 2.1 in [10] is a filtered homotopy.

\section{§3. The filtered Poincaré lemma}

We will explain here how the results of [10], Section 4 extend to the case of transversal $m$-crystals.

Let $(S, \mathfrak{a}, \mathfrak{b})$ be a $m$-PD-scheme with $p$ locally nilpotent and $p \in \mathfrak{a}$. Let $X$ be an $S$-scheme to which the $m$-PD-structure of $S$ extends. We will consider the crystalline site of level $m$ of $X / S$ that was introduced in Section 4.1 of $[9]$ and the corresponding topos $(X / S)_{\text {cris }}^{(m)}$.

Unless otherwise specified, we will assume in this section that $X$ is smooth over $S$. 
Notations 3.1. If $Y$ is any object in a topos $\mathcal{T}$, we will denote by $\mathcal{T}_{\mid Y}$ the localized category and by $j_{Y}: \mathcal{T}_{\mid Y} \rightarrow \mathcal{T}$ the restriction map.

In particular, we will consider here the localization $(X / S)_{\operatorname{cris} \mid X}^{(m)}$ at the trivial immersion of $X$ in itself and the restriction morphism

$$
j_{X}:(X / S)_{\text {cris } \mid X}^{(m)} \longrightarrow(X / S)_{\text {cris }}^{(m)} \text {. }
$$

By definition, we have $\left(j_{X *} \mathcal{F}\right)_{(U, T)}=\mathcal{F}_{(U, T \times U)}$ and we see in this case that $j_{X *}$ is exact.

We will also consider the projection

$$
u_{X}:(X / S)_{\text {cris }}^{(m)} \longrightarrow X_{Z a r}
$$

on the Zariski site, given by $\Gamma\left(U, u_{X *} \mathcal{F}\right)=\Gamma\left((U / S)_{\text {cris }}^{(m)}, \mathcal{F}\right)$. Note that this is not a morphism of ringed topoi because there is no natural map $\mathcal{O}_{X} \rightarrow u_{X *} \mathcal{O}_{X / S}^{(m)}$. However, the composite

$$
u_{\mid X}:=u_{X} \circ j_{X}:(X / S)_{\text {cris } \mid X}^{(m)} \longrightarrow X_{Z a r}
$$

is in a natural way a morphism of ringed topoi because it is nothing but the realization morphism: $u_{\mid X *} \mathcal{F}=\mathcal{F}_{X}$. We also see that $u_{\mid X *}$ is an exact functor.

Details can be found in Chapter 5 of [4] for the $m=0$ case. The generalization is straightforward.

Proposition 3.2. With the above notations,

1. The ideal $\mathcal{K}_{X / S}^{(m)}:=j_{X *} j_{X}^{-1} \mathcal{I}_{X / S}^{(m)}$ of $j_{X *} j_{X}^{-1} \mathcal{O}_{X / S}^{(m)}$ is an m-PD-ideal.

2. There is a canonical exact sequence

$$
0 \longrightarrow \mathcal{K}_{X / S}^{(m)} \longrightarrow j_{X *} j_{X}^{-1} \mathcal{O}_{X / S}^{(m)} \longrightarrow u_{X}^{-1} \mathcal{O}_{X} \longrightarrow 0 .
$$

3. For all $k \in \mathbf{Z}$, we have $\mathcal{K}_{X / S}^{(m)}{ }^{\{k\}}=j_{X *} j_{X}^{-1}\left(\mathcal{I}_{X / S}^{(m)}{ }^{\{k\}}\right)$.

Proof. One easily checks that, if $\mathcal{F}$ is any $\mathcal{O}_{X}$-module, the adjunction map

$$
j_{X *} j_{X}^{-1} u_{X}^{-1} \mathcal{F} \longrightarrow u_{X}^{-1} \mathcal{F}
$$


is an isomorphism. In particular, if we apply the exact functor $j_{X *} j_{X}^{-1}$ to the exact sequence

$$
0 \longrightarrow \mathcal{I}_{X / S}^{(m)} \longrightarrow \mathcal{O}_{X / S}^{(m)} \longrightarrow u_{X}^{-1} \mathcal{O}_{X} \longrightarrow 0
$$

we get the expected exact sequence

$$
0 \longrightarrow j_{X *} j_{X}^{-1} \mathcal{I}_{X / S}^{(m)} \longrightarrow j_{X *} j_{X}^{-1} \mathcal{O}_{X / S}^{(m)} \longrightarrow u_{X}^{-1} \mathcal{O}_{X} \longrightarrow 0
$$

Thus, we see that if $U \subset X$ is any open subset and $U \hookrightarrow Y$ an $m$-PDthickening, we have the following exact sequence

$$
0 \longrightarrow\left(K_{X / S}^{(m)}\right)_{Y} \longrightarrow \mathcal{O}_{P} \longrightarrow \mathcal{O}_{U} \longrightarrow 0
$$

where $P:=P_{U m}\left(Y \times_{S} X\right)$. Hence, we see that $\left(K_{X / S}^{(m)}\right)_{Y}$ is the $m$-PD-ideal $\mathcal{I}_{P}$ of $P$. It follows that $K_{X / S}^{(m)}$ is an $m$-PD-ideal and that for all $k \in \mathbf{Z}$, $\left(K_{X / S}^{(m)\{k\}}\right)_{Y}=\mathcal{I}_{P}^{\{k\}}=\left(\mathcal{I}_{X / S}^{(m){ }^{\{k\}}}\right)_{Y}$

Definition 3.3. If $\mathcal{F}$ is a filtered $\mathcal{O}_{X}$-module, its linearization (of level $m$ ) is $L^{(m)}(\mathcal{F}):=j_{X *} u_{\mid X}^{*} \mathcal{F}$ endowed with the saturation of the filtration $L^{(m)}\left(\mathrm{Fil}^{k} \mathcal{F}\right)$ with respect to the $m$-PD-ideal $\mathcal{K}_{X / S}^{(m)}{ }^{\{\bullet\}}$ of $L^{(m)}\left(\mathcal{O}_{X}\right):=$ $j_{X *} j_{X}^{-1} \mathcal{O}_{X / S}^{(m)}$.

LEMMA 3.4. If $\mathcal{F}$ is a filtered $\mathcal{O}_{X}$-module, we have a canonical isomorphism of filtered modules

$$
L^{(m)}(\mathcal{F})_{X}=\mathcal{P}_{X / S m}(1) \otimes_{\mathcal{O}_{X}} \mathcal{F}
$$

Proof. Thanks to Proposition 4.3 (1) of [10], only the assertion concerning the filtration has to be checked. Since saturation is just tensor product with the ideal filtration, it is sufficient to note that, as in the proof of Lemma $3.2,\left(\mathcal{K}_{X / S}^{(m)}\right)_{X}=\mathcal{I}_{X / S m}(1)$.

We recall that $L^{(m)}\left(\Omega_{X / S m}^{\bullet}\right)$ has a natural structure of complex of crystals whose realization on $X$ is nothing but the linearized de Rham complex $L_{X}\left(\Omega_{X / S m}^{\bullet}\right)$. Thanks to the lemma, this is compatible with the filtrations.

We can now state the formal Poincaré lemma in its crystalline form. 
THEOREM 3.5. If $E$ is a filtered $\mathcal{O}_{X / S}^{(m)}$-module which is saturated with respect to $\mathcal{I}_{X / S}^{(m)}\{\bullet\}$, then the morphism

$$
E \longrightarrow E \otimes_{\mathcal{O}_{X / S}^{(m)}} L^{(m)}\left(\Omega_{X / S m}^{\bullet}\right)
$$

is a true filtered quasi-isomorphism. More precisely, locally on $\operatorname{Cris}^{(m)}(X / S)$, it is a filtered homotopy equivalence.

Proof. Since the question is local, we may assume, thanks to Proposition 2.10, that the canonical map $\mathcal{O}_{X} \rightarrow L_{X}\left(\Omega_{X / S m}^{\bullet}\right)$ is a filtered homotopy equivalence.

Let's start with the case $E=\mathcal{O}_{X / S}^{(m)}$. We have to check that, for any any $m$-PD-thickening $U \hookrightarrow T$, the morphism

$$
\mathcal{O}_{T} \longrightarrow L^{(m)}\left(\Omega_{X / S m}^{\bullet}\right)_{T}
$$

is locally on $T$ a filtered homotopy equivalence. Thus, we may assume that $U \hookrightarrow T$ has a retraction. Since the pull-back of a filtered homotopy is still a filtered homotopy, we obtain a filtered homotopy equivalence on $T$ from the one on $X$ using this retraction.

In general, tensoring with $E$ gives a morphism

$$
E \otimes_{\mathcal{O}_{X / S}^{(m)}} \mathcal{O}_{X / S}^{(m)} \longrightarrow E \otimes_{\mathcal{O}_{X / S}^{(m)}} L^{(m)}\left(\Omega_{X / S m}^{\bullet}\right)
$$

which is locally a filtered homotopy equivalence. Since the filtration on $E$ is saturated, the canonical identification

$$
E \otimes_{\mathcal{O}_{X / S}^{(m)}} \mathcal{O}_{X / S}^{(m)}=E
$$

is compatible with the filtrations. The proof is therefore complete.

The definition of a transversal $m$-crystal is given in [9], Section 4 . Roughly speaking, it is a crystal of transversal modules, but the reader should consider looking at the above reference if he really wants a precise definition as well as a description of its relation with Griffiths transversality.

Proposition 3.6. Let $E$ be a transversal m-crystal on $X / S$. If $\mathcal{F}$ is any filtered $\mathcal{O}_{X}$-module, there is a canonical isomorphism of filtered modules

$$
E \otimes L^{(m)}(\mathcal{F}) \simeq L^{(m)}\left(E_{X} \otimes \mathcal{F}\right)
$$


Proof. We already have an isomorphism of crystals thanks to Proposition 4.3 (3) of [10]. More precisely, if If $U \subset X$ is any open subset and $U \hookrightarrow Y$ an $m$-PD-thickening, we have an isomorphism

$$
E_{Y} \otimes \mathcal{O}_{P} \otimes \mathcal{F} \simeq E_{P} \otimes \mathcal{F} \simeq \mathcal{O}_{P} \otimes E_{Y} \otimes \mathcal{F}
$$

where $P:=P_{U m}\left(Y \times_{S} X\right)$ as in the proof of Proposition 3.2, and we need to show that it is compatible with filtrations. But this follows from the definition in [9], 4.2.2 of a transversal $m$-crystal.

Corollary 3.7. If $E$ is a transversal m-crystal on $X / S$, there is a canonical true filtered quasi-isomorphism

$$
E \longrightarrow L^{(m)}\left(E_{X} \otimes_{\mathcal{O}_{X}} \Omega_{X / S m}^{\bullet}\right)
$$

More precisely, locally on $\operatorname{Cris}^{(m)}(X / S)$, it is a filtered homotopy equivalence.

In order to obtain the filtered Poincaré lemma at the cohomological level, we need the following:

Proposition 3.8. If $\mathcal{F}$ is a filtered $\mathcal{O}_{X}$-module, we have a canonical isomorphism in the filtered derived category $R u_{X *} L^{(m)}(\mathcal{F})=\mathcal{F}$.

Proof. We must show that, for all $k \in \mathbf{Z}$, we have $R^{i} u_{X *} \operatorname{Fil}^{k} L^{(m)}(\mathcal{F})=$ 0 for $i>0$ and $u_{X *} \operatorname{Fil}^{k} L^{(m)}(\mathcal{F})=\mathrm{Fil}^{k} \mathcal{F}$.

By definition, the filtration of $L^{(m)}(\mathcal{F})$ is the saturation of the filtration $L^{(m)}\left(\mathrm{Fil}^{k} \mathcal{F}\right)$ with respect to the $m$-PD-ideal $\mathcal{K}_{X / S}^{(m)}{ }^{\{\bullet}$ :

$$
\operatorname{Fil}^{k} L^{(m)}(\mathcal{F})=\sum_{i+j=k, i \geq 0} \mathcal{K}_{X / S}^{(m)}{ }^{\{i\}} L^{(m)}\left(\mathrm{Fil}^{j} \mathcal{F}\right)
$$

Using Proposition 3.2.3, we obtain

$$
\operatorname{Fil}^{k} L^{(m)}(\mathcal{F})=\sum_{i+j=k, i \geq 0}\left(j_{X *} j_{X}^{-1} \mathcal{I}_{X / S}^{(m)}{ }^{\{i\}}\right)\left(j_{X *} u_{\mid X}^{*}\left(\mathrm{Fil}^{j} \mathcal{F}\right)\right)
$$

Since $j_{X *}$ is exact, it follows that $\mathrm{Fil}^{k} L^{(m)}(\mathcal{F})=j_{X *} \mathcal{E}_{k}$ with

$$
\mathcal{E}_{k}:=\sum_{i+j=k, i \geq 0} j_{X}^{-1} \mathcal{I}_{X / S}^{(m)}{ }^{\{i\}} u_{\mid X}^{*}\left(\mathrm{Fil}^{j} \mathcal{F}\right)
$$


Regarding the higher direct images, it is sufficient to recall that, since $j_{X *}$ and $u_{\mid X *}$ are exact, we have

$$
R^{i} u_{X *} \operatorname{Fil}^{k} L^{(m)}(\mathcal{F})=R^{i} u_{X *} j_{X *} \mathcal{E}_{k}=R^{i} u_{\mid X *} \mathcal{E}_{k}=0
$$

for $i>0$.

Now, we know from Proposition 4.3 (2) of [10] that, for any $\mathcal{O}_{X}$-module $\mathcal{F}$, we have $u_{X *} L^{(m)}(\mathcal{F})=\mathcal{F}$. Also, $u_{X}^{-1}$ being fully faithful, $u_{X *} u_{X}^{-1} \mathcal{F}=$ $\mathcal{F}$. Since $u_{X *}$ is left exact and $\mathcal{K}_{X / S}^{(m)}$ is the kernel of the natural map $L^{(m)}\left(\mathcal{O}_{X}\right) \rightarrow u_{X}^{-1} \mathcal{O}_{X}$, we see that $u_{X *} \mathcal{K}_{X / S}^{(m)}=0$. It follows that $u_{X *}$ ignores saturation with respect to $\mathcal{K}_{X / S}^{(m)}$ and so,

$$
u_{X *} \operatorname{Fil}^{k} L^{(m)}(\mathcal{F})=u_{X *} L^{(m)}\left(\mathrm{Fil}^{k} \mathcal{F}\right)=\mathrm{Fil}^{k} \mathcal{F}
$$

as asserted.

TheOREM 3.9. If $E$ is a transversal $m$-crystal on $X / S$, there is a canonical isomorphism in the filtered derived category

$$
R u_{X *} E \simeq E_{X} \otimes \Omega_{X / S m}^{\bullet}
$$

Proof. Using Proposition 3.8, this is an direct consequence of 3.7.

COROllary 3.10. Even if we no longer assume $X$ smooth, but if $i$ : $X \hookrightarrow Y$ is an embedding into a smooth $S$-scheme and if $E$ is a transversal $m$-crystal on $X$, then there is a canonical isomorphism in the filtered derived category

$$
i_{*} R u_{X *} E \simeq\left(i_{\text {cris } *} E\right)_{Y} \otimes \Omega_{Y / S m}^{\bullet}
$$

Proof. Works exactly as at the end of Section 4 in [10].

COROLlaRY 3.11. In the situation of the previous corollary, there is a canonical filtered isomorphism

$$
\left.R \Gamma\left((X / S)_{\mathrm{cris}}^{(m)}, E\right) \simeq R \Gamma\left(Y,\left(i_{\mathrm{cris}, m *} E\right)_{Y} \otimes \Omega_{Y / S m}^{\bullet}\right)\right) .
$$

Therefore, for all $i$, we have

$$
H_{\mathrm{cris}, m}^{i}\left(X, \mathrm{Fil}^{k} E\right)=H^{i}\left(\mathrm{Fil}^{k}\left(\left(i_{\mathrm{cris}, m *} E\right)_{Y} \otimes \Omega_{Y / S m}^{\bullet}\right)\right) .
$$




\section{$\S 4$. Differentials of higher level on a group scheme}

In this section, which is independent of the previous one, we define the conormal complex of level $m$ of a group scheme and study invariant differential forms of higher level.

Definition 4.1. The simplicial object $G^{g r}(\bullet)$ associated to a group $G$ in a category with products has components $G^{g r}(r):=G^{r}$, differentials $d_{i}: G^{r+1} \rightarrow G^{r}$ defined by

$$
\begin{aligned}
d_{0}\left(g_{1}, \ldots, g_{r+1}\right) & =\left(g_{2}, \ldots, g_{r+1}\right) \\
& \vdots \\
d_{i}\left(g_{1}, \ldots, g_{r+1}\right) & =\left(g_{1}, \ldots, g_{i-1}, g_{i} g_{i+1}, g_{i+2}, \ldots, g_{r+1}\right) \\
& \vdots \\
d_{r+1}\left(g_{1}, \ldots, g_{r+1}\right) & =\left(g_{1}, \ldots, g_{r}\right)
\end{aligned}
$$

and degeneracy arrows $s_{i}: G^{r-1} \rightarrow G^{r}$ defined by

$$
s_{i}\left(g_{1}, \ldots, g_{r-1}\right)=\left(g_{1}, \ldots, g_{i}, 1, g_{i+1}, \ldots, g_{r-1}\right) .
$$

Lemma 4.2. If $G$ is a group in a category with products, the maps

$$
G^{r+1} \longrightarrow G^{r}:\left(g_{1}, \ldots, g_{r+1}\right) \longmapsto\left(g_{1}^{-1} g_{2}, g_{2}^{-1} g_{3}, \ldots, g_{r}^{-1} g_{r+1}\right)
$$

define a morphism of simplicial objects $\nu(\bullet): G^{\text {prod }}(\bullet) \rightarrow G^{g r}(\bullet)$.

Proof. This is easily checked.

Let $G$ be a group scheme over a scheme $S, p_{G}: G \rightarrow S$ its structural morphism, $1_{G}: S \hookrightarrow G$ its unit section and

$$
\mu, p_{1}, p_{2}: G \times{ }_{S} G \longrightarrow G
$$

the group law and the projections.

Definition 4.3. The conormal complex of level $m$ of $G$ is the Berthelot-Lieberman filtered complex $\omega_{G m}^{\bullet}$ associated to the unit embedding of $S$ into $G^{g r}(\bullet)$.

It will be convenient to write $P_{1 m}(\bullet)$ for the $m$-PD-envelope of the unit section in $G^{g r}(\bullet)$ and modify the other notations accordingly. 
Remark 4.4. As in the case of the de Rham complex, for which the results are recalled in Remark 2.5, we have a very simple local description of the situation in the case of a smooth group scheme. If $s_{1}, \ldots, s_{n}$ is a regular sequence of local parameters for the unit section, then $\mathcal{P}_{1 m}(r)$ is a free module on generators $s^{\left\{J_{1}\right\}} \otimes \cdots \otimes s^{\left\{J_{r}\right\}}$ with $\left|J_{i}\right| \geq 0$ and $\mathcal{I}_{1 m}^{\{k\}}(r)$ is generated by the $s^{\left\{J_{1}\right\}} \otimes \cdots \otimes s^{\left\{J_{r}\right\}}$ with $\left|J_{1}\right|+\cdots+\left|J_{r}\right| \geq k$. Thus, if $\bar{s}_{i}$ denotes the image of $s_{i}$ in $\omega_{G m}^{1}$, we see that $\omega_{G m}^{1}$ is a free module on generators $\bar{s}^{J}$ with $0<|J| \leq p^{m}$ and that, for bigger $r, \omega_{G m}^{r}$ is generated by the $\bar{s}^{J_{1}} \otimes \cdots \otimes \bar{s}^{J_{r}}$ with $0<\left|J_{i}\right| \leq p^{m}$, subject to some relations. Of course, the $k$-th step of the Hodge filtration $\mathrm{Fil}^{k} \omega_{G m}^{r}$ has the same generators subject to the additional condition that $\left|J_{1}\right|+\cdots+\left|J_{r}\right| \geq k$. In particular, $\mathrm{Fil}^{r} \omega_{G m}^{r}=\omega_{G m}^{r}$.

Proposition 4.5. The morphism $\nu(\bullet)$ of Lemma 4.2 induces morphisms of filtered complexes

$$
\nu(\bullet): \mathcal{P}_{1 m}(\bullet) \longrightarrow p_{G *} \mathcal{P}_{G m}(\bullet)
$$

and

$$
\nu^{\bullet}: \omega_{G m}^{\bullet} \longrightarrow p_{G *} \Omega_{G m}^{\bullet}
$$

Proof. This follows from the functoriality of the construction of the Berthelot-Lieberman complex.

Remark 4.6. Of course, we also have for each $r$ morphisms of filtered modules

$$
\nu(r): p_{G}^{*} \mathcal{P}_{1 m}(r) \longrightarrow \mathcal{P}_{G m}(r)
$$

and

$$
\nu^{r}: p_{G}^{*} \omega_{G m}^{r} \longrightarrow \Omega_{G m}^{r} .
$$

There are also morphisms of filtered complexes

$$
\mathcal{P}_{1 m}(\bullet)=1_{G}^{*} p_{G}^{*} \mathcal{P}_{1 m}(\bullet) \longrightarrow 1_{G}^{*} p_{G}^{*} p_{G *} \mathcal{P}_{G m}(\bullet) \longrightarrow 1_{G}^{*} \mathcal{P}_{G m}(\bullet)
$$

and in particular

$$
\omega_{G m}^{\bullet} \longrightarrow 1_{G}^{*} \Omega_{G m}^{\bullet}
$$

Without assuming that $G$ is smooth, we cannot really say more.

If $g \in G(S)$, we denote by $T_{g}: G \rightarrow G$ the left translation map given by $h \mapsto g h$. 
Definition 4.7. A section $\xi$ of $\mathcal{P}_{G m}(r)$, or $\mathcal{P}_{G m}^{k}(r)$, is invariant by translation by $g \in G(S)$ if $T_{g}^{*}(\xi)=\xi$. Moreover, $\xi$ is invariant by translation if it is invariant by translation by any section of $G_{S^{\prime}}$ for any extension $S^{\prime} \rightarrow S$ of the basis.

We indicate with a superscript $\bullet{ }^{i n v}$ the subsheaf of translation invariant sections.

\section{Proposition 4.8.}

1. If $G$ is smooth, then for all $r, \nu(r)$ is a canonical isomorphism of filtered modules

$$
p_{G}^{*} \mathcal{P}_{1 m}(r) \simeq \mathcal{P}_{G m}(r)
$$

and it follows that

$$
p_{G}^{*} \omega_{G m}^{r} \simeq \Omega_{G m}^{r} .
$$

2. The morphism $\nu(\bullet)$ gives also isomorphisms of filtered complexes

$$
\mathcal{P}_{1 m}(\bullet) \simeq 1_{G}^{*} \mathcal{P}_{G m}(\bullet) \simeq \mathcal{P}_{G m}^{i n v}(\bullet)
$$

and

$$
\omega_{G m}^{\bullet} \simeq 1_{G}^{*} \Omega_{G m}^{\bullet} \simeq \Omega_{G m}^{\bullet i n v} .
$$

Proof. Note first that the diagram

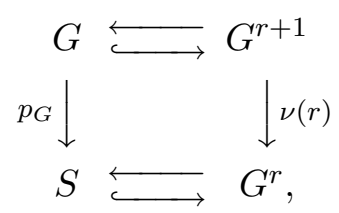

where the top (resp. bottom) arrows are the first projection (resp. structural map $p_{G}$ ) and the diagonal embedding (resp. unit section $1_{G}$ ), is cartesian both ways. Since $G$ is smooth, it follows from the second assertion of Proposition 2.1 that, for all $r, \nu(r)$ induces an isomorphism of filtered modules

$$
p_{G}^{*} \mathcal{P}_{1 m}(r) \simeq \mathcal{P}_{G m}(r)
$$

Since the Berthelot-Lieberman complex is obtained as a quotient of the $\mathrm{Fil}^{1}$ by the differential ideal generated by the $\mathrm{Fil}^{p^{m}+1}$, we obtain also an isomorphism

$$
p_{G}^{*} \omega_{G m}^{r} \simeq \Omega_{G m}^{r}
$$

for each $r$. 
It follows from the first part that the morphism of filtered complexes

$$
\mathcal{P}_{1 m}(\bullet) \longrightarrow 1_{G}^{*} \mathcal{P}_{G m}(\bullet)
$$

of Remark 4.6 is an isomorphism. We also have injective maps

$$
\mathcal{P}_{1 m}(r) \longleftrightarrow p_{G *} \mathcal{P}_{G m}(r) .
$$

Moreover, since for $g \in G(S)$ we have

$$
\nu(r) \circ\left(T_{g} \times \cdots \times T_{g}\right)=\nu(r): G^{r+1} \longrightarrow G^{r},
$$

we see that the image of $\mathcal{P}_{1 m}(\bullet)$ in $p_{G *} \mathcal{P}_{G m}(\bullet)$ is contained in $\mathcal{P}_{G m}^{i n v}(\bullet)$. We want to show that the map

$$
\mathcal{P}_{1 m}(\bullet) \longleftrightarrow \mathcal{P}_{G m}^{i n v}(\bullet)
$$

is bijective. Since

$$
p_{G *} \mathcal{P}_{G m}(r) \simeq p_{G *} \mathcal{O}_{G} \otimes_{\mathcal{O}_{S}} \mathcal{P}_{1 m}(r)
$$

and $G$ is flat, we are reduced to showing that the canonical map $\mathcal{O}_{S} \rightarrow \mathcal{O}_{G}^{\text {inv }}$ is bijective. If we consider translation by the the identity

$$
G \times G \longrightarrow G \times G, \quad(g, h) \longmapsto(g, g h),
$$

we see that, if $f$ is a section of $\mathcal{O}_{G}^{i n v}$, then $\mu^{*}(f)=p_{1}^{*}(f)$. Pulling back by $\left(1_{G} \times \operatorname{Id}_{G}\right)^{*}$, we get $f=p_{G}^{*} 1_{G}^{*}(f)$ which is what we want.

Proposition 4.9. If $G$ is a smooth abelian group scheme and if we let

$$
\delta:=p_{2}^{*}-\mu^{*}+p_{1}^{*}: p_{G *} \mathcal{P}_{G m}(1) \longrightarrow p_{G^{2} *} \mathcal{P}_{G^{2} m}(1),
$$

then the morphism $\nu(1)$ induces an isomorphism of filtered modules

$$
p_{G *} \mathcal{I}_{G m}(1) \cap \operatorname{ker} \delta=\mathcal{I}_{G m}^{i n v}(1) \cap \operatorname{ker} d
$$

and therefore also

$$
p_{G *} \Omega_{G m}^{1} \cap \operatorname{ker} \delta=\Omega_{G m}^{1 i n v} \cap \operatorname{ker} d .
$$


Proof. If $g \in G(S)$ and $i_{g}: G \rightarrow G \times G, h \mapsto(g, h)$, we have $p_{2} \circ i_{g}=$ $\operatorname{Id}_{G}, \mu \circ i_{g}=T_{g}$ and $p_{1} \circ i_{g}=g \circ p_{G}$. It follows that

$$
i_{g}^{*} \circ \delta=\left(\mathrm{Id}-T_{g}^{*}\right)+p_{G}^{*} \circ g^{*} .
$$

If $\xi \in p_{G *} \mathcal{I}_{G m}(1)$ then, trivially, $g^{*}(\xi)=0$ and it follows that $i_{g}^{*}(\delta(\xi))=$ $\xi-T_{g}^{*}(\xi)$. Thus, any $\xi \in p_{G *} \mathcal{I}_{G m}(1) \cap \operatorname{ker} \delta$ is $g$-invariant. This is true for any $g$ and after any base change. It follows that

$$
p_{G *} \mathcal{I}_{G m}(1) \cap \operatorname{ker} \delta \subset \mathcal{I}_{G m}^{i n v}(1) .
$$

Since $G$ is a commutative group scheme, the diagrams

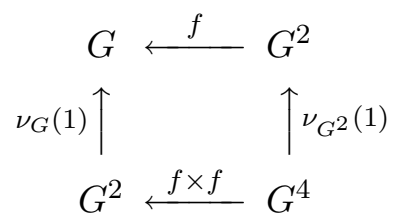

are all commutative for $f=p_{1}, f=p_{2}$ and $f=\mu$. Observe that $\mathcal{P}_{1_{G^{2}} m}(1)=$ $\mathcal{P}_{1 m}(2)$. By functoriality, the diagram

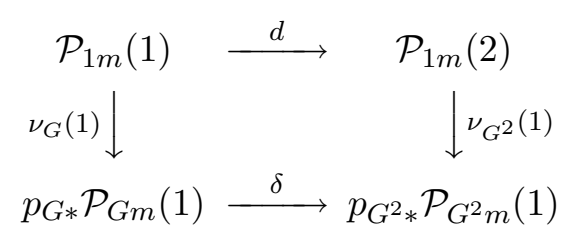

is also commutative.

Since we assumed that $G$ is smooth, the vertical arrows induce filtered isomorphisms with the invariant part in the bottom and we obtain

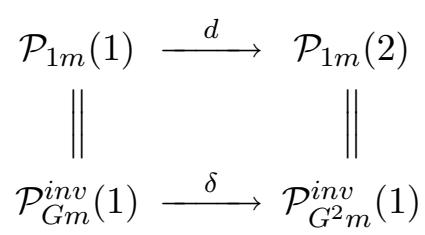

from which it follows that $d=\delta$ on $P_{G m}^{i n v}(1)$ and the result follows.

Notations 4.10. The sheaf

$$
p_{G *} \Omega_{G m}^{1} \cap \operatorname{ker} \delta=\Omega_{G m}^{1 i n v} \cap \operatorname{ker} d \simeq \omega_{G m}^{1} \cap \operatorname{ker} d
$$

of closed invariant forms will be generally written $\omega_{G m, 0}$. Notice that, since the first differential of the complex $\omega_{G m}^{\bullet}$ is just the zero map, we also have $\omega_{G m, 0} \simeq \mathcal{H}^{1}\left(\omega_{G m}^{\bullet}\right)$. 
When $m=0$, in which case we drop $m$ from the notations, the situation is a lot simpler since invariant forms are automatically closed, and in particular, the kernel of $\delta$ is exactly the sheaf of invariant differential forms. In general, the group inclusions $i_{1}, i_{2}: G \rightarrow G \times G$ induce morphisms $i_{k}^{*}: \omega_{G^{2} m}^{1} \rightarrow \omega_{G m}^{1}$ and the composite maps

$$
\omega_{G m}^{1} \stackrel{\delta}{\longrightarrow} \omega_{G^{2} m}^{1} \stackrel{i_{k}^{*}}{\longrightarrow} \omega_{G m}^{1}
$$

are zero. When $m=0, \omega_{G}^{1}$ is nothing but the conormal sheaf to the unit section and we have an isomorphism $\left(i_{1}^{*}, i_{2}^{*}\right): \omega_{G^{2}}^{1} \simeq \omega_{G}^{1} \oplus \omega_{G}^{1}$. It follows that $\delta=0$ on $\omega_{G}^{1}$. The first example below already shows that this is not necessarily the case when $m>0$.

\section{EXAMPLES 4.11.}

1. If $G=\mathbf{G}_{a S}$ with parameter $t$, then $\omega_{G m}^{1}$ is the free $\mathcal{O}_{S}$-module on $\bar{t}, \bar{t}^{2}, \ldots, \bar{t}^{m}$. The group law is given by $t \mapsto t \otimes 1+1 \otimes t$ and it follows that

$$
\delta\left(t^{k}\right)=t^{k} \otimes 1-(t \otimes 1+1 \otimes t)^{k}+1 \otimes t^{k}=-\sum_{i=1}^{k-1}\left(\begin{array}{c}
k \\
i
\end{array}\right) t^{i} \otimes t^{k-i}
$$

In particular, we have $\delta(t)=0$ and therefore, we always have $\bar{t} \in$ $\omega_{G m, 0}$.

But we also see that, if char $S=p$ (where we mean $p \mathcal{O}_{S}=0$ ), then $\bar{t}^{p^{j}} \in \omega_{G m, 0}$ for all $j$. Actually, in this case, $\omega_{G m, 0}$ is free on the generators $\bar{t}, \bar{t}^{p}, \ldots \bar{t}^{p^{m}}$. In particular, we see that the filtration on $\omega_{G m, 0}$ can have length exactly $p^{m}-1$. We also see that when $p \neq 2$, then $\vec{t}^{2}$ is not closed.

In the case $S=\operatorname{Spec} \mathbf{Z} / 4$ (so that $p=2$ ) and $m=1$, we see that $\delta\left(\bar{t}^{2}\right)=-2 \bar{t} \otimes \bar{t} \neq 0$ but that $2 \bar{t}^{2} \in \omega_{G 1,0}$. Thus, $\omega_{G m, 0}$ is not always locally free.

The opposite map in $\mathbf{G}_{a}$ is given by $t \mapsto-t$ and the difference is therefore given by $t \mapsto-t \otimes 1+1 \otimes t$. It follows that the canonical inclusion $\omega_{G m}^{1} \hookrightarrow p_{*} \Omega_{G m}^{1}$ sends $\bar{t}$ to $d t$ and therefore, $d t$ is a closed invariant differential of level $m$ on $\mathbf{G}_{a S}$. On the other hand, for $m>0$, $(d t)^{2}$ is an invariant differential form which is not closed in general. 
2. If $G=\mathbf{G}_{m S}$ with parameter $t$, then $\omega_{G m}^{1}$ is the free module on $\bar{s}, \bar{s}^{2}, \ldots, \bar{s}^{p^{m}}$ with $s=t-1$. Let us consider $\log t^{p^{m}}:=\log (1+s)^{p^{m}} \in$ $\widehat{\mathcal{P}_{1 m}(1)}$. Using the fact that the group law is given by $t \mapsto t \otimes t$, it is not difficult to check that $\delta\left(\log t^{p^{m}}\right)=0$. Actually, this is a purely formal calculation that can be done over $\mathbf{C}$ where this is well known. It follows that

$$
\sum_{i=1}^{p^{m}}(-1)^{i} \frac{p^{m}}{i} \bar{s}^{i} \in \omega_{G m, 0} .
$$

Note that, unlike the case $m=0, \mathrm{Fil}^{2} \omega_{G m, 0} \neq 0$ in general. For example, if $p=2, m=1$ and $S=\operatorname{Spec} \mathbf{Z} / 4$, then we have $2 \bar{s}-\bar{s}^{2} \in \mathrm{Fil}^{1}$ and $2 \bar{s}^{2} \in \mathrm{Fil}^{2}$.

Since the inverse on $\mathbf{G}_{m}$ is given by $t \mapsto t^{-1}$, the difference is given by $t \mapsto t^{-1} \otimes t$. Thus we see that $t-1$ is sent to $t^{-1} \otimes t-1 \otimes 1=t^{-1}(1 \otimes$ $t-t \otimes 1)$ and it follows that the canonical inclusion $\omega_{G m}^{1} \hookrightarrow p_{*} \Omega_{G m}^{1}$ sends $\bar{s}$ to $d t / t$. Finally, we get that

$$
\sum_{i=1}^{p^{m}}(-1)^{i} \frac{p^{m}}{i}\left(\frac{d t}{t}\right)^{i}
$$

is a closed invariant differential of level $m$ on $\mathbf{G}_{m S}$.

3. We assume now $p \neq 2$ and consider the Legendre elliptic curve $E$ given by the equation

$$
y^{2}=x(x-1)(x-\lambda)
$$

over $\mathbf{A}_{S}^{1} \backslash\{0,1\}$. Since we are interested in the behavior at $O$ which is the point at infinity, we make the usual change of coordinates $z=$ $-x / y, w=-1 / y$ and the equation becomes

$$
w=z(z-w)(z-\lambda w)
$$

Thus, $P_{1 m}(1)$ is the $m$-th divided power envelope of $\mathcal{O}_{\mathbf{A}_{S}^{1} \backslash\{0,1\}}[z]$ with respect to $z$ and $\omega_{E m}^{1}$ is the free module on $\bar{z}, \ldots, \bar{z}^{p^{m}}$. A quick calculation as explained in Chapter 4 of [12], for example, shows that the group law is given by

$$
z \longmapsto 1 \otimes z+z \otimes 1+(\lambda+1)\left(z \otimes z^{2}+z^{2} \otimes z\right)+\cdots .
$$

Assume now that $p=3$ and $m=1$. Then, $\omega_{E 1}^{1}$ is the free $\mathcal{O}_{S}$-module on $\bar{z}, \bar{z}^{2}, \bar{z}^{3}$ and $\omega_{E 1}^{2}$ is generated by the $\bar{z}^{i} \otimes \bar{z}^{j}$ for $1 \leq i, j \leq 3$ subject 
to the relations

$$
\begin{gathered}
\bar{z}^{3} \otimes \bar{z}^{3}=0, \\
\bar{z}^{2} \otimes \bar{z}^{3}+\bar{z}^{3} \otimes \bar{z}^{2}=0, \\
2 \bar{z} \otimes \bar{z}^{3}+3 \bar{z}^{2} \otimes \bar{z}^{2}+2 \bar{z}^{3} \otimes \bar{z}=0 .
\end{gathered}
$$

Taking into account the symmetry of the series above due to the commutativity of the group law, one sees that higher powers don't play any role and that

$$
\begin{gathered}
\delta(\bar{z})=-(\lambda+1)\left(\bar{z} \otimes \bar{z}^{2}+\bar{z}^{2} \otimes \bar{z}\right), \\
\delta\left(\bar{z}^{2}\right)=-2 \bar{z} \otimes \bar{z}-(\lambda+1)\left(\bar{z}^{2} \otimes \bar{z}^{2}\right), \\
\delta\left(\bar{z}^{3}\right)=-3\left(\bar{z} \otimes \bar{z}^{2}+\bar{z}^{2} \otimes \bar{z}\right) .
\end{gathered}
$$

It turns out that $-3 \bar{z}+(1+\lambda) \bar{z}^{3}$ is in $\omega_{E 1,0}$.

Assume moreover that char $S=3$. Then we get $(1+\lambda) \bar{z}^{3}$ which is in $\mathrm{Fil}^{3}$. It follows from the formulas that $\omega_{E 1,0}$ is actually free of rank 1 with generator $\bar{z}^{3}$ outside $\lambda=-1$. However, at the fiber $\lambda=-1$, both $\bar{z}$ and $\bar{z}^{3}$ are in $\omega_{E 1,0}$. In other words, the supersingular fiber is characterized by the fact that $\omega_{E 1,0}$ is of rank 2 in contrast with the general case where it has rank 1. This is a phenomenon that is specific to higher level because in the classical situation, $\omega_{E 0,0}=\omega_{E}$ is globally free of rank 1 . Note however that in the case $m=1$ and $p=3$, then $F i l^{p^{m}} \omega_{E m, 0}$ is also globally free of rank 1 .

The next question would be to describe the canonical inclusion $\omega_{E m}^{1} \hookrightarrow$ $p_{*} \Omega_{E m}^{1}$. Since taking opposite on $E$ is given by horizontal symmetry, it sends $z$ to $-z$, and it follows that the difference in $E$ is given by

$$
z \longmapsto 1 \otimes z-z \otimes 1+(\lambda+1)\left(-z \otimes z^{2}+z^{2} \otimes z\right)+\cdots
$$

When $m=0$, one can check that $\bar{z}$ is sent to $d x / 2 y$ but we have been too lazy to do the computations in other cases.

Proposition 4.12. If $A$ is an abelian scheme over $S$, the $E_{2}$-term of the Hodge to de Rham spectral sequence of level $m$ of $A / S$ is

$$
E_{2}^{i j}=R^{j} p_{A *} \mathcal{O}_{A} \otimes_{\mathcal{O}_{S}} \mathcal{H}^{i}\left(\omega_{A m}^{\bullet}\right)
$$


Proof. Consider the Hodge to de Rham spectral sequence of level $m$ :

$$
E_{1}^{i j}=R^{j} p_{A *} \Omega_{A m}^{i} \Rightarrow \mathcal{H}_{d R m}^{n}(A / S)
$$

We know that, for all $i, \Omega_{A m}^{i}=p_{A}^{*} \omega_{A m}^{i}$. Since the sheaves $\omega_{A m}^{i}$ are locally free, the projection formula tells us that

$$
R^{j} p_{A *} \Omega_{A m}^{i}=R^{j} p_{A *} \mathcal{O}_{A} \otimes_{\mathcal{O}_{S}} \omega_{A m}^{i}
$$

and we get the spectral sequence

$$
E_{2}^{i j}=R^{j} p_{A *} \mathcal{O}_{A} \otimes_{\mathcal{O}_{S}} \mathcal{H}^{i}\left(\omega_{A m}^{\bullet}\right) \Rightarrow \mathcal{H}_{d R m}^{n}(A / S) .
$$

Corollary 4.13. The sequence

$$
0 \longrightarrow \omega_{A m, 0} \longrightarrow \mathcal{H}_{d R m}^{1}(A / S) \longrightarrow \mathcal{R}^{1} p_{A *} \mathcal{O}_{A}
$$

is (left) exact.

Proof. Just note that $\omega_{A m, 0}=H^{1}\left(\omega_{A m}^{\bullet}\right)$.

Remark 4.14. When $S$ is smooth, using some results from [13], we can show that we actually get a short exact sequence. We hope to be able to prove this in general in a forthcoming article.

\section{§5. Crystalline extension groups}

In this section, we generalize to higher level the first results of the second chapter of [3]. The point is to describe the "mod $p$ filtration on crystalline Dieudonné modules of level $m$ " (Proposition 5.14). The situation is way more complicated than in the classical $m=0$ case because the filtration has more than one step and not all invariant forms are closed.

Definitions 5.1. A category $I$ is said very small if $\mathrm{Ob}(I)$ is countable and for each $\alpha, \beta \in \operatorname{Ob}(I), \operatorname{Hom}(\alpha, \beta)$ is finite. A decoration on a very small category $I$ is a pair comprising a degree map $d: \mathrm{Ob}(I) \rightarrow \mathbf{Z}$ with finite fibers and a sign map $\epsilon: \operatorname{Arr}(I) \rightarrow\{ \pm\}$.

Notations 5.2. If $Y$ is any object in a topos $\mathcal{T}$, we will denote by $\mathbf{Z}^{(Y)}$ the free abelian group on $Y$ in $\mathcal{T}$. 
Let $(I, d, \epsilon)$ be a decorated category and $Y_{\bullet}:=\left(Y_{\alpha}, f_{\lambda}\right)$ a diagram indexed by $I$ in $\mathcal{T}$. We set

$$
C_{n}\left(Y_{\bullet}\right):=\bigoplus_{d(\alpha)=n} \mathbf{Z}^{\left(Y_{\alpha}\right)}
$$

and let $\delta_{n}: C_{n} \rightarrow C_{n-1}$ be given by

$$
\sum_{\lambda: \alpha \rightarrow \beta} \epsilon(\lambda) f_{\lambda}: \mathbf{Z}^{\left(Y_{\alpha}\right)} \longrightarrow \mathbf{Z}^{\left(Y_{\beta}\right)}
$$

whenever $\operatorname{deg}(\alpha)=n$ and $\operatorname{deg}(\beta)=n-1$.

Definition 5.3. A diagram $Y_{\bullet}$ indexed by a decorated category $(I, d, \epsilon)$ is nice if $C\left(Y_{\bullet}\right)$ is a complex.

Remark 5.4. P. Deligne has shown that any abelian group $G$ in a topos $\mathcal{T}$ has a canonical left resolution $C_{\bullet}(G)$ that fits in the above setting. More precisely, there exists a decorated category $I$ and a nice diagram $\Delta(G)_{\bullet}:=$ $\left(G^{n_{\alpha}}, f_{\lambda}\right)$ indexed by $I$ in the subcategory of $\mathcal{T}$ generated by the powers of $G$, the projections and the group law such that $C_{\bullet}(G)=C_{\bullet}(\Delta(G) \bullet)$.

To the best of our knowledge, this result of Deligne is unpublished, and we can offer no better reference than what is said in the remark at the end of 2.1.5 of [3]. We have taken the option of using this Deligne construction because we think it is the most elegant and natural way to state Theorem 5.12. For readers who fill uncomfortable using unpublished results, we should mention that our main application (Proposition 5.14) can be deduced from a weaker version of the theorem (Remark 5.13.1) that relies only on a truncated resolution as in [3], Chapter 2, Section 1. The point is that Proposition 5.14 involves only the first three terms of the Deligne resolution that we now describe.

The diagram has only one object $G$ in degree 0 . In degree 1 , there is also a unique object $G^{2}$ and the morphisms $G^{2} \rightarrow G$ are

$$
\begin{array}{rlrr}
G^{2} & \longrightarrow & G & \text { sign } \\
\left(g_{1}, g_{2}\right) & \longmapsto & g_{1} & - \\
\left(g_{1}, g_{2}\right) & \longmapsto & g_{1}+g_{2} & + \\
\left(g_{1}, g_{2}\right) & \longmapsto & g_{2} & -
\end{array}
$$


In degree 2 , there are two objects, namely $G^{2}$ and $G^{3}$. The morphisms from degree 2 to degree 1 are given by

$$
\begin{array}{ccc}
G^{2} & \longrightarrow G^{2} & \text { sign } \\
\left(g_{1}, g_{2}\right) & \longrightarrow\left(g_{1}, g_{2}\right) & + \\
\left(g_{1}, g_{2}\right) & \longrightarrow\left(g_{2}, g_{1}\right) & -
\end{array}
$$

and by

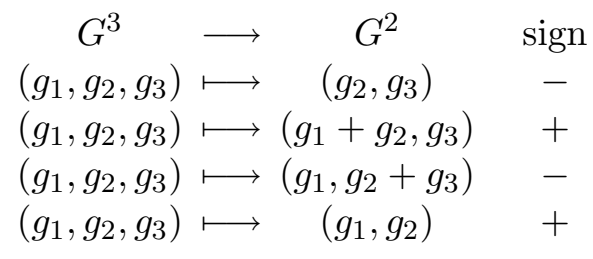

Thus we get

$$
\mathbf{Z}^{\left(G^{3}\right)} \oplus \mathbf{Z}^{\left(G^{2}\right)} \longrightarrow \mathbf{Z}^{\left(G^{2}\right)} \longrightarrow \mathbf{Z}^{(G)}
$$

and the maps are given by

$$
\left[g_{1}, g_{2}\right] \longmapsto-\left[g_{1}\right]+\left[g_{1}+g_{2}\right]-\left[g_{2}\right]
$$

from degree 1 to degree 0 and

$$
\begin{aligned}
{\left[g_{1}, g_{2}\right] } & \longmapsto\left[g_{1}, g_{2}\right]-\left[g_{2}, g_{1}\right] \\
{\left[g_{1}, g_{2}, g_{3}\right] } & \longmapsto-\left[g_{2}, g_{3}\right]+\left[g_{1}+g_{2}, g_{3}\right]-\left[g_{1}, g_{2}+g_{3}\right]+\left[g_{1}, g_{2}\right]
\end{aligned}
$$

from degree 2 to degree 1.

LEMma 5.5. Let $Y_{\bullet}$ be a nice diagram indexed by a decorated category $(I, d, \epsilon)$ in some topos $\mathcal{T}$ and $E$ an abelian sheaf in $\mathcal{T}$. Then, there is a spectral sequence

$$
E_{1}^{r, s}=\bigoplus_{d(\alpha)=r} R^{s} j_{Y_{\alpha} *} j_{Y_{\alpha}}^{-1} E \Rightarrow \mathcal{E} x t^{r+s}\left(C\left(Y_{\bullet}\right), E\right) .
$$

Proof. If $Y$ is any object of $\mathcal{T}$, we have

$$
\mathcal{H o m}_{G r}\left(\mathbf{Z}^{(Y)}, E\right)=\mathcal{H o m}(Y, E)=j_{Y *} j_{Y}^{-1} E .
$$

It follows that the complex $\mathcal{H} m_{G r}\left(C_{\bullet}\left(Y_{\bullet}\right), E\right)$ is canonically isomorphic to a complex whose terms are all of the form $\bigoplus_{\operatorname{deg} \alpha=n} j_{Y_{\alpha} *} j_{Y_{\alpha}}^{-1} E$. Since pulling back by localization is exact and preserves injective sheaves, we get the spectral sequence by applying this remark to an injective resolution of $E$. 
Let $(S, \mathfrak{a}, \mathfrak{b})$ be an $m$-PD-scheme with $p$ locally nilpotent and $p \in \mathfrak{a}$.

Let $X$ be an $S$-scheme to which the $m$-PD-structure of $S$ extends.

We denote by $\mathrm{Sch}_{/ X}^{\prime}$ the category of $X$-schemes to which the $m$-PDstructure of $S$ extends. The Zariski topology on this category is coarser than the canonical topology and we obtain an embedding of $\operatorname{Sch}_{/ X}^{\prime}$ into the corresponding topos $X_{\mathrm{ZAR}^{\prime}}$. We will now use the big crystalline topos of level $m,(X / S)_{\mathrm{CRIS}}^{(m)}$ that was introduced in [7] (but considering only schemes to which the $m$-PD structure extends). Composing the embedding $\mathrm{Sch}_{/ X}^{\prime} \hookrightarrow X_{\mathrm{ZAR}^{\prime}}$ with the canonical map

$$
v_{X / S *}: X_{\mathrm{ZAR}^{\prime}} \longrightarrow(X / S)_{\mathrm{CRIS}}^{(m)}
$$

from Section 1.10 of [7] gives a functor

$$
\begin{aligned}
\operatorname{Sch}^{\prime} X & \longrightarrow(X / S)_{\mathrm{CRIS}}^{(m)} \\
Y & \longmapsto \underline{Y}
\end{aligned}
$$

Remark 5.6. Note that if $Y \in \mathrm{Sch}_{/ X}^{\prime}$ with structural morphism $f_{Y}$ : $Y \rightarrow X$ then the canonical morphism

$$
f_{Y / X}:(Y / S)_{\mathrm{CRIS}}^{(m)} \longrightarrow(X / S)_{\mathrm{CRIS}}^{(m)}
$$

factors as an isomorphism $(Y / S)_{\mathrm{CRIS}}^{(m)} \simeq(X / S)_{\mathrm{CRIS} \mid \underline{Y}}^{(m)}$ followed by the localization map

$$
j_{\underline{Y}}:(X / S)_{\mathrm{CRIS} \mid \underline{Y}}^{(m)} \longrightarrow(X / S)_{\mathrm{CRIS}}^{(m)} .
$$

Proposition 5.7. Let $Y_{\bullet}$ be a nice diagram in $\mathrm{Sch}_{/ X}^{\prime}$ indexed by a decorated category $(I, d, \epsilon)$. If $E$ is an abelian sheaf on $\operatorname{CRIS}^{(m)}(X / S)$, there is a spectral sequence

$$
E_{1}^{r, s}=\bigoplus_{d(\alpha)=r} R^{s} f_{Y_{\alpha} / X *} f_{Y_{\alpha} / X}^{-1} E \Rightarrow \mathcal{E} x t^{r+s}\left(C\left(\underline{Y}_{\bullet}\right), E\right) .
$$

Proof. Taking into account the previous remark, this immediately follows from Lemma 5.5.

Remarks 5.8. Just as in Chapter III, Section 4 of [1], if $Y \in \operatorname{Sch}_{X}^{\prime}$, there is a morphism of topoi

$$
(Y / S)_{\mathrm{cris}}^{(m)} \longrightarrow(X / S)_{\mathrm{CRIS}}^{(m)}
$$


whose inverse image functor $E \mapsto E_{Y}$ might be called restriction. For any sheaf $E$ on $\operatorname{CRIS}^{(m)}(X / S)$ and any morphism $g: Y^{\prime} \rightarrow Y$, there is a canonical transition map $g^{-1} E_{Y} \rightarrow E_{Y^{\prime}}$ and these data uniquely determine $E$. An $m$-crystal on the big site can be defined as an $\mathcal{O}_{X / S^{(m)}}^{\text {-module }}$ $E$ such that all $E_{Y}$ are $m$-crystals and the transition maps induce isomorphisms $g^{*} E_{Y} \simeq E_{Y^{\prime}}$. In particular, the functor $E \mapsto E_{X}$ is an equivalence of categories between $m$-crystals on $\operatorname{CRIS}^{(m)}(X / S)$ and $m$-crystals on $\operatorname{Cris}^{(m)}(X / S)$. Finally, note that any filtered (resp. transversal) $\mathcal{O}_{X / S^{-}}^{(m)}$ module $\left(E, \mathrm{Fil}^{\bullet}\right)$ on $\mathrm{CRIS}^{(m)}(X / S)$ restricts for each $Y$ to a filtered (resp. transversal) $\mathcal{O}_{Y / S}^{(m)}$-module $\left(E_{Y}, \mathrm{Fil}^{\bullet} E_{Y}\right)$ on $\mathrm{Cris}^{(m)}(Y / S)$.

For future reference, note also that, as for level 0, in which case this is proved in 1.1.16.4 of [3], we have for any abelian sheaf on $\operatorname{CRIS}^{(m)}(Y / S)$,

$$
\left(R f_{Y / X *} E\right)_{(U, T)}=R f_{U \times_{X} Y / T *} E_{U \times_{X} Y} .
$$

Definition 5.9. A big transversal $m$-crystal on $X / S$ is a crystal $E$ on $\operatorname{CRIS}^{(m)}(X / S)$, endowed with a filtration Fil ${ }^{\bullet}$ such that for each $Y \in \operatorname{Sch}_{/ X}^{\prime}$, the filtered $m$-crystal $\left(E_{Y}, \mathrm{Fil}^{\bullet} E_{Y}\right)$ is a transversal $m$-crystal.

Lemma 5.10. Let $Y \in \mathrm{Sch}_{/ X}^{\prime}$ and $(U \hookrightarrow T) \in \mathrm{CRIS}^{(m)}(X / S)$. Let $i: U \times_{X} Y \hookrightarrow Z$ be a closed immersion into a smooth $Z$ in $\mathrm{Sch}_{/ T}^{\prime}$. If $E$ is a big transversal $m$-crystal on $X / S$, we have a canonical isomorphism

$$
\left(R^{s} f_{Y / X *} f_{Y / X}^{-1} \mathrm{Fil}^{k} E\right)_{(U, T)}=R^{s} f_{Z *} \operatorname{Fil}^{k}\left[\left(i_{\text {cris } *} E_{U \times_{X} Y}\right)_{Z} \otimes \Omega_{Z / T m}^{\bullet}\right] .
$$

Proof. First of all, Corollary 3.10 tells us that

$$
\mathrm{Fil}^{k} i_{*} R u_{Y *} E_{U \times_{X} Y} \simeq \operatorname{Fil}^{k}\left[\left(i_{\mathrm{cris} *} E_{U \times_{X} Y}\right)_{Z} \otimes \Omega_{Z / T m}^{\bullet}\right] .
$$

Actually, since we work with filtered derived categories and $i_{*}$ is exact, we have

$$
\mathrm{Fil}^{k} i_{*} R u_{Y *} E_{U \times_{X} Y}=i_{*} R u_{Y *} \mathrm{Fil}^{k} E_{U \times_{X} Y}
$$

and therefore

$$
i_{*} R u_{Y *} \mathrm{Fil}^{k} E_{U \times_{X} Y} \simeq \operatorname{Fil}^{k}\left[\left(i_{\mathrm{cris} *} E_{U \times_{X} Y}\right)_{Z} \otimes \Omega_{Z / T m}^{\bullet}\right] .
$$

Since $i_{*}$ is exact, applying $R f_{Z *}$ gives

$$
R f_{U \times_{X} Y / T *} \operatorname{Fil}^{k} E_{U \times_{X} Y} \simeq R f_{Z *} \operatorname{Fil}^{k}\left[\left(i_{\text {cris } *} E_{U \times_{X} Y}\right)_{Z} \otimes \Omega_{Z / T m}^{\bullet}\right]
$$


and, taking cohomology, we obtain

$$
R^{s} f_{U \times_{X} Y / T *} \operatorname{Fil}^{k} E_{U \times_{X} Y} \simeq R^{s} f_{Z *} \operatorname{Fil}^{k}\left[\left(i_{\text {cris } *} E_{U \times_{X} Y}\right)_{Z} \otimes \Omega_{Z / T m}^{\bullet}\right] .
$$

Finally, Remark 5.8 tells us that

$$
\left(R^{s} f_{Y / X *} f_{Y / X}^{-1} \mathrm{Fil}^{k} E\right)_{(U, T)}=R^{s} f_{U \times_{X} Y / T *} \mathrm{Fil}^{k} E_{U \times_{X} Y} .
$$

Proposition 5.11. Let $Y_{\bullet}$ be a nice diagram in $\mathrm{Sch}_{/ X}^{\prime}$ and $(U \hookrightarrow T) \in$ $\operatorname{CRIS}^{(m)}(X / S)$. Let $Z_{\bullet}$ be a nice diagram in $\mathrm{Sch}_{/ T}^{\prime}$ with all $Z_{\alpha}$ smooth and $i_{\bullet}: U \times_{X} Y_{\bullet} \hookrightarrow Z_{\bullet}$ a compatible family of closed immersions. If $E$ is a big transversal $m$-crystal on $X / S$, we have a canonical spectral sequence

$$
\begin{aligned}
& E_{1}^{r, s}=\bigoplus_{d(\alpha)=r} R^{s} f_{Z_{\alpha} *} \operatorname{Fil}^{k}\left[\left(i_{\alpha \text { cris } *} E_{U \times_{X} Y_{\alpha}}\right)_{Z_{\alpha}} \otimes \Omega_{Z_{\alpha} / T m}^{\bullet}\right] \\
& \Rightarrow \mathcal{E} x t^{r+s}\left(C\left(\underline{Y}_{\bullet}\right), \mathrm{Fil}^{k} E\right)_{(U, T)} .
\end{aligned}
$$

Proof. Since taking value on some object $(U, T)$ is an exact functor, we know from Proposition 5.7 that there is a spectral sequence

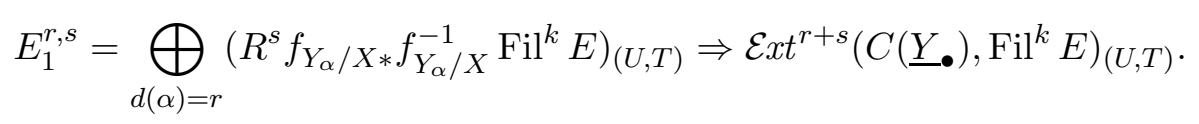

It is therefore sufficient to prove that for each $\alpha$, we have

$$
\left(R^{s} f_{Y_{\alpha} / X *} f_{Y_{\alpha} / X}^{-1} \operatorname{Fil}^{k} E\right)_{(U, T)}=R^{s} f_{Z_{\alpha} *} \operatorname{Fil}^{k}\left[\left(i_{\alpha \operatorname{cris} *} E_{U \times_{X} Y_{\alpha}}\right)_{Z_{\alpha}} \otimes \Omega_{Z_{\alpha} / T m}^{\bullet}\right]
$$

and this follows from the lemma.

Applying this to the nice diagram $\Delta(G)$ • of Remark 5.4, that gives rise to Deligne resolution, we immediately get

THEOREM 5.12. Let $G$ be an abelian group scheme in $\mathrm{Sch}_{/ X}^{\prime}$ and $(U \hookrightarrow$ $T) \in \operatorname{CRIS}^{(m)}(X / S)$. Let $H$ be a smooth abelian group scheme on $T$ and $i: G_{U} \hookrightarrow H_{U}$ an immersion of groups. If $E$ is a big transversal $m$-crystal on $X / S$, we have a canonical spectral sequence

$$
\begin{aligned}
E_{1}^{r, s}=\bigoplus_{d(\alpha)=r} R^{s} f_{H^{n_{\alpha} *}} \operatorname{Fil}^{k}\left[\left(i_{\alpha \mathrm{cris} *} E_{G_{U}^{n_{\alpha}}}\right)_{H^{n_{\alpha}}}\right. & \otimes \Omega_{H^{n_{\alpha} / T m}}^{\bullet} \\
& \Rightarrow \mathcal{E} x t^{r+s}\left(\underline{G}, \mathrm{Fil}^{k} E\right)_{(U, T)} .
\end{aligned}
$$


Remarks 5.13.

1. If we use the truncated complex, we obtain a spectral sequence

$$
E_{1}^{r, s}=\bigoplus_{d(\alpha)=r} R^{s} f_{H^{n_{\alpha} *}} \operatorname{Fil}^{k}\left[\left(i_{\alpha \operatorname{cris} *} E_{G_{U}^{n_{\alpha}}}\right)_{H^{n_{\alpha}}} \otimes \Omega_{H^{n_{\alpha}} / T m}^{\bullet}\right] \Rightarrow \mathcal{H}^{r+s}
$$

with

$$
\mathcal{H}^{i}=\mathcal{E} x t^{i}\left(\underline{G}, \mathrm{Fil}^{k} E\right)_{(U, T)} \quad \text { for } i \leq 1 .
$$

2. If $E$ is any $m$-crystal on $X / S$, then the trivial transversal filtration $\mathcal{I}_{X / S}^{\{k\}} E$ turns it into a big transversal $m$-crystal and both Proposition 5.11 and Theorem 5.12 apply. Also, the theorem is still valid if we replace $G$ with a complex of abelian groups.

3. When $H$ is affine, if we denote by $\left[K^{\bullet \bullet}\right]_{\text {sing }}$ the simple complex associated to a bicomplex $K^{\bullet \bullet}$, we have, as in Theorem 2.1.8 of [3], an isomorphism in the derived category

$$
\begin{aligned}
\operatorname{Fil}^{k}\left[\bigoplus_{d(\alpha)=\bullet} f_{H^{n_{\alpha} *}}\left[\left(i_{\alpha \operatorname{cris} *} E_{G_{U}^{n_{\alpha}}}\right)_{H^{n_{\alpha}}} \otimes \Omega_{H^{n_{\alpha}} / T m}^{\bullet}\right]_{\operatorname{sing}}\right] \\
\\
\simeq R \mathcal{H o m}\left(\underline{G}, \mathrm{Fil}^{k} E\right)_{(U, T)} .
\end{aligned}
$$

The proof goes exactly as in [3].

Proposition 5.14. Let $G$ be a smooth abelian group scheme in $\mathrm{Sch}_{/ X}$ and $U \in \mathrm{Sch}_{/ X}^{\prime}$. If $k>0$, we have

$$
\begin{aligned}
& \mathcal{H o m}\left(\underline{G}, \mathcal{I}_{X / S}^{\{k\}}\right)_{(U, U)}=\quad 0 \\
& \mathcal{E}_{x t^{1}}\left(\underline{G}, \mathcal{I}_{X / S}^{\{k\}}\right)_{(U, U)}=F i l^{k} \omega_{G_{U} m, 0}
\end{aligned}
$$

where $\omega_{G_{U} m, 0}$ denotes, as in 4.10 , the sheaf of closed invariant forms of level $m$ on $G_{U}$. In particular,

$$
\mathcal{E}_{x t^{1}}\left(\underline{G}, \mathcal{I}_{X / S}\right)_{(U, U)}=\omega_{G_{U}, 0} .
$$

Proof. We consider the spectral sequence of Theorem 5.12 (see also Remark 5.13.1)

$$
E_{1}^{r, s}=\bigoplus_{d(\alpha)=r} R^{s} f_{G_{U}^{n_{\alpha}} *} \mathrm{Fil}^{k} \Omega_{G_{U}^{n_{\alpha}} / U m}^{\bullet} \Rightarrow \mathcal{E} x t^{p+q}\left(\underline{G}, \mathcal{I}_{X / S}^{\{k\}}\right)_{(U, U)}
$$


Note first that $E_{1}^{r, s}=0$ for $r<0$ or $s<0$. Actually, since $k>0$, we have Fil $^{k} \mathcal{O}_{G_{U} n_{\alpha}}=0$ and it follows that $E_{1}^{r, s}=0$ for $s=0$ also. Therefore

$$
\mathcal{H o m}\left(\underline{G}, \mathcal{I}_{X / S}^{\{k\}}\right)_{(U, U)}=0
$$

and

$$
\mathcal{E} x t^{1}\left(\underline{G}, \mathcal{I}_{X / S}^{\{k\}}\right)_{(U, U)}=E_{2}^{0,1}=\operatorname{ker} d_{1}^{0,1}: E_{1}^{0,1} \longrightarrow E_{1}^{1,1} .
$$

Recall that if $K^{\bullet}$ is any complex with $K^{i}=0$ for $i<s$ and $f$ a left exact functor, then

$$
R^{s} f_{*} K^{\bullet}=R^{s} f_{*} \operatorname{ker} d^{s}[-s]=f_{*} \operatorname{ker} d^{s}=\operatorname{ker} f_{*} d^{s}
$$

Thus, here, we have for all $\alpha$,

$$
\begin{aligned}
& R^{1} f_{G_{U}^{n_{\alpha} *}} \mathrm{Fil}^{k} \Omega_{G_{U}^{n_{\alpha}} / U m}^{\bullet} \\
& =\operatorname{ker}\left[d: f_{G_{U}^{n_{\alpha} *}} \mathrm{Fil}^{k} \Omega_{G_{U}^{n_{\alpha}} / U m}^{1} \rightarrow f_{G_{U}^{n_{\alpha}}} \mathrm{Fil}^{k} \Omega_{G_{U}^{n_{\alpha}} / U m}^{2}\right] .
\end{aligned}
$$

Since filtration commutes with kernel and direct image, we have

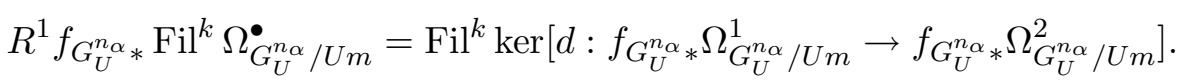

It follows from the description of the first terms of the Deligne resolution that

$$
\begin{aligned}
& E_{1}^{0,1}=\mathrm{Fil}^{k} \operatorname{ker}\left[d: f_{G_{U^{*}}} \Omega_{G_{U} / U m}^{1} \rightarrow f_{G_{U^{*}}} \Omega_{G_{U} / U m}^{2}\right], \\
& E_{1}^{1,1}=\mathrm{Fil}^{k} \operatorname{ker}\left[d: f_{G_{U^{*}}^{2}} \Omega_{G_{U}^{2} / U m}^{1} \rightarrow f_{G_{U}^{2}{ }^{*}} \Omega_{G_{U}^{2} / U m}^{2}\right]
\end{aligned}
$$

and that

$$
d_{1}^{0,1}=-p_{1}^{*}+\mu^{*}-p_{2}^{*}=-\delta
$$

with the notations of Proposition 4.9. Hence, we obtain

$$
\begin{aligned}
E_{2}^{0,1}=\mathrm{Fil}^{k} \operatorname{ker}\left[d: f_{G_{U^{*}}} \Omega_{G_{U} / U m}^{1}\right. & \left.\rightarrow f_{G_{U^{*}}} \Omega_{G_{U}^{\prime} U m}^{2}\right] \\
\cap \operatorname{ker}\left[\delta: f_{G_{U} *} \Omega_{G_{U} / U m}^{1}\right. & \left.\rightarrow f_{G_{U}^{2}}^{2} \Omega_{G_{U}^{2} / U m}^{1}\right]
\end{aligned}
$$

which is exactly $F i l^{k} \omega_{G_{U} m, 0}$. 


\section{REFERENCES}

[1] P. Berthelot, Cohomologie crystalline des schémas de caractéristique $p>0$, Lecture Notes in Mathematics, vol. 407, Springer-Verlag, Berlin, 1974.

[2] P. Berthelot, $\mathcal{D}$-modules arithmétiques I. Opérateurs différentiels de niveau fini, Ann. Sci. École Norm. Sup. (4), 29 (1996), 185-272.

[3] P. Berthelot, L. Breen and W. Messing, Théorie de Dieudonné crystalline II, Lecture Notes in Mathematics, vol. 930, Springer-Verlag, Berlin, 1982.

[4] P. Berthelot and A. Ogus, Notes on crystalline cohomology, Princeton University Press, 1978.

[5] R. Crew, Crystalline cohomology of singular varieties, Geometric aspects of Dwork theory. Vol. I, Walter de Gruyter GmbH \& Co. KG, Berlin, 2004, pp. 451-462.

[6] P. Deligne, Théorie de Hodge II, Inst. Hautes Études Sci. Publ. Math., (40) (1971), $5-57$.

[7] J.-Y. Étesse and B. Le Stum, Fonctions L associées aux F-isocristaux surconvergents. II. Zéros et pôles unités, Invent. Math., 127 (1997), 1-31.

[8] L. Illusie, Complexe cotangent et déformations I, Lecture Notes in Mathematics, vol. 239, Springer-Verlag, Berlin, 1971.

[9] B. Le Stum and A. Quirós, Transversal crystals of finite level, Ann. Inst. Fourier, 47 (1997), 69-100.

[10] B. Le Stum and A. Quirós, The exact Poincaré lemma in crystalline cohomology of higher level, J. Algebra, 240 (2001), 559-588.

[11] A. Ogus, F-crystals, Griffiths transversality, and the Hodge decomposition, Astérisque, 221 (1994).

[12] J. H. Silverman, The arithmetic of elliptic curves, Graduate Texts in Mathematics, vol. 106, Springer-Verlag, New York, 1986.

[13] F. Trihan, Fonction L unité d'un groupe de Barsotti-Tate, Manuscripta Math., 96 (1998), 397-419.

Bernard Le Stum

IRMAR, Université de Rennes I

Campus de Beaulieu

F-35042 Rennes Cedex

France

bernard.le-stum@univ-rennes1.fr

Adolfo Quirós

Departamento de Matemáticas

Universidad Autónoma de Madrid

E-28049 Madrid

Spain

adolfo.quiros@uam.es 